\title{
Prenatal allergen and diesel exhaust exposure and their effects on allergy in adult offspring mice
}

\author{
Lin Corson', Huaijie Zhu1', Chunli Quan², Gabriele Grunig1,2, Manisha Ballaney1, Ximei Jin², Frederica P Perera³, \\ Phillip H Factor ${ }^{1}$, Lung-Chi Chen² and Rachel L Miller*1,2
}

\begin{abstract}
Background: Multiple studies have suggested that prenatal exposure to either allergens or air pollution may increase the risk for the development of allergic immune responses in young offspring. However, the effects of prenatal environmental exposures on adult offspring have not been well-studied. We hypothesized that combined prenatal exposure to Aspergillus fumigatus (A. fumigatus) allergen and diesel exhaust particles will be associated with altered IgE production, airway inflammation, airway hyperreactivity (AHR), and airway remodeling of adult offspring.

Methods: Following sensitization via the airway route to A. fumigatus and mating, pregnant BALB/C mice were exposed to additional A. fumigatus and/or diesel exhaust particles. At age 9-10 weeks, their offspring were sensitized and challenged with A. fumigatus.

Results: We found that adult offspring from mice that were exposed to A. fumigatus or diesel exhaust particles during pregnancy experienced decreases in IgE production. Adult offspring of mice that were exposed to both A. fumigatus and diesel exhaust particles during pregnancy experienced decreases in airway eosinophilia.
\end{abstract}

Conclusion: These results suggest that, in this model, allergen and/or diesel administration during pregnancy may be associated with protection from developing systemic and airway allergic immune responses in the adult offspring.

\section{Background}

Epidemiological studies and murine models suggest that prenatal environmental exposures can enhance the risk for developing asthma in the offspring [1,2]. In humans, prenatal exposures to air pollutants such as environmental tobacco smoke (ETS) and polycyclic aromatic hydrocarbons (PAHs) have been shown to be associated with asthma-related outcomes in young children $[1,3,4]$. In mice, prenatal exposure to residual oil fly ash was associated with increased airway hyperresponsiveness, allergic inflammation, and elevated immunoglobulin (Ig) $\mathrm{E}$ and $\mathrm{IgG}_{1}$ in the ovalbumin (OVA) sensitized offspring by age 16-37 days [2]. Offspring mice of mothers that were exposed to diesel exhaust particles (DEP) and immunologically inert substances such as titanium dioxide and carbon black particles during pregnancy also were more susceptible to developing airway hyperreactivity and

\footnotetext{
* Correspondence: rlm14@columbia.edu

${ }^{1}$ Division of Pulmonary, Allergy and Critical Care Medicine, Department of Medicine, Columbia University College of Physicians and Surgeons, New York, New York 10032, USA

Full list of author information is available at the end of the article
}

inflammation following ovalbumin sensitization, suggesting that the mechanism to induce enhanced risk for asthma by inert substance exposure is not antigen-specific [5]. Most recently, a diet high in methyl donors during pregnancy was associated with a greater degree of airway allergic inflammation that was transmitted to a third generation of mice. These changes were associated with altered DNA methylation of Runt-related transcription factor 3 (RUNX3), implicating epigenetic regulation in the transmission of an asthma-related phenotype across generations[6].

Alternately, some prenatal exposures have induced protection from the asthma phenotype. Lipopolysaccharide (LPS or endotoxin) administered prenatally to mice led to the development of lower anti-OVA IgE and $\operatorname{IgG}_{1}$ levels, eosinophilia in BAL fluid, and reduced phorbol 12myristate 13-acetate (PMA), inomycin, and OVAinduced $\mathrm{T}$ helper (Th) 2 cytokine production in the offspring $[7,8]$. In epidemiological studies, prenatal exposure to farms, sources of endotoxin exposure, was associated as well with childhood protection from 
asthma, hay fever, and atopic sensitization [9]. Furthermore, mice whose mothers were immunized with Dermatophagoides pteronyssinus (D. pteronyssinus) allergen prior to mating developed significant decreases in total and anti-D. pteronyssinus IgE, $\operatorname{IgG}_{1}, \operatorname{IgG}_{2 \mathrm{a}}$ and $\mathrm{IgG}_{2 \mathrm{~b}}$ levels upon resensitization in comparison to offspring of unexposed mice [10]. Hence, in some models, prenatal allergen exposure may confer immunological tolerance or protection from atopy in the offspring.

Despite these advances, many key questions still need to be elucidated. These include questions about the effects of airborne prenatal exposures to toxins of concern in the urban environment, as well as their possible long-term adverse effects on adult offspring. Our objective was to determine the effects of concomitant and chronic aerosolized prenatal exposure to allergen and diesel exhaust particles, two environmental exposures implicated in inner city asthma [11,12], on phenotypes that develop in adult offspring mice. Our strategy was to employ the $A$. fumigatus mouse model that induces strong allergic responses via the airway route in the absence of adjuvants and, hence, arguably better mimics clinical asthma [13]. Diesel exhaust was routed through an exposure chamber and administered during pregnancy $[14,15]$. We hypothesized that combined prenatal exposure to A. fumigatus and diesel exhaust particles would be associated with altered IgE, airway inflammation, airway hyperreactivity (AHR), and airway remodeling in adult mice offspring.

\section{Methods}

\section{A. fumigatus sensitization}

Six week old wild-type female and male BALB/c mice were obtained from Jackson Laboratories (Bar Harbor, ME). Males and females were housed separately prior to mating. All animals were housed at New York University (NYU) animal facility (Tuxedo, NY) and fed a commercial pellet mouse feed. Mice were lightly anesthetized with isoflurane ( $2 \%$ inhaled). Intranasal application of $A$. fumigatus (62.5 ug) (Hollister-Stier Co., Spokane, WA; measured endotoxin dose $<0.16 \mathrm{EU} / \mathrm{ml}$ : Endotoxin Testing Service, Cambrex Bio Science Walkersville, Inc, MD) in $50 \mathrm{ul}$ of saline or saline vehicle alone was administered five times, four days apart, beginning 20 days prior to mating. Pregnant mice were treated again with $A$. fumigatus or saline on day 7 and 14 after mating. Offspring were separated from their mothers at 21 days of age. At 9-10 weeks of age, all offspring were treated with either five or six dosages of $A$. fumigatus each dose four days apart (Figure 1). All experimental procedures were approved by IACUCs at Columbia University and New York University.

\section{Diesel exposure}

Diesel exhaust was produced by a 5500-watt single cylinder diesel engine generator (Yanmar YDG 5500EE-6EI; Osaka, Japan) that contained a 418-cc displacement engine (Model LE100EE-DEGY6), as described [15,16]. The engine was operated at a maximum engine load condition using Number 2 on-road ultra-low-sulfur diesel fuel delivered from a local gas station (SOS Fuels, Tuxedo Park, NY) and 15W/40 engine oil (SAE, 15W/40, Delo400, Chevron Products Company, San Ramon, CA). The diesel exhaust particles (DEP) were diluted to a desirable level through a serial dilution system with HEPA-filtered ambient air, and routed to a $1 \mathrm{~m}^{3}$ flow-through exposure chamber where mice were exposed. Pregnant mice were exposed for 5 hours (average 5.18 hours) a day, Mondays through Fridays, to DEP or HEPA (high efficient particle) filtered ambient air (as negative control) in parallel during the second and third weeks of pregnancy (Figure 1).

The mass concentrations of the DEP in the exposure chamber were recorded every 20 minutes using a realtime Personal DataRam (PDR) aerosol monitor (Model: PDR1000, MIE Inc., Bedford, MA). DEP also were collected daily onto Teflon filters (Gelman Teflo, $37 \mathrm{~mm}, 0.2$ $u$ m pore; Gelman Sciences, Ann Arbor, MI) for subsequent gravimetric analyses. Particle size distributions were measured with a Wide-Range Particle Spectrometer (0.01 to $10 \mu \mathrm{m}$, WPS, MSP Corp., Shoreview, MN). The average particle concentration was $1.09 \mathrm{mg} / \mathrm{m}^{3}$. The DEP atmosphere had a count median aerodynamic diameter of $80 \mathrm{~nm}$, and a mass median aerodynamic diameter of $152 \mathrm{~nm}$

\section{Blood collection and measurement of $\lg \mathrm{E}_{1} \lg \mathrm{G}_{1}, \lg \mathrm{G}_{2 \mathrm{a}}$}

Sera were obtained from adult female mice immediately prior to the first dose of A. fumigatus, and 2 days following the fifth dose of A. fumigatus versus saline prior to mating. Sera were obtained from their offspring prior to the first, and one day after the third, fifth, and sixth (last) dose of A. fumigatus. Sera were aliquoted and frozen. Total Ig levels were measured by ELISA using isotype specific capture antibodies for IgE, $\operatorname{IgG}_{1}$ and $\operatorname{IgG}_{2 \mathrm{a}}(\mathrm{BD}$ PharMingen, Franklin Lakes, NJ), following a previously described protocol [17]. Briefly, 96 well microtiter plates were coated with rat anti-mouse IgE, $\operatorname{IgG}_{1}$ or $\operatorname{IgG}_{2 \mathrm{a}}$. Sera were diluted 1:20 for IgE, 1:10,000 for $\operatorname{IgG}_{1}$, and 1:100 for $\mathrm{IgG}_{2 \mathrm{a}}$. Biotin labeled rat-mouse IgE, $\operatorname{IgG}_{1}$ and $\mathrm{IgG}_{2 \mathrm{a}}$ along with AKP (alkaline-phosphatase) Streptavidan (BD Pharmingen, Franklin Lakes, NJ) were used for detection. Specimens were run in duplicate and averaged.

\section{BAL and cellular analysis}

Mice were euthanized at median age 12.5 weeks and bronchoalveolar lavage was performed three times on 


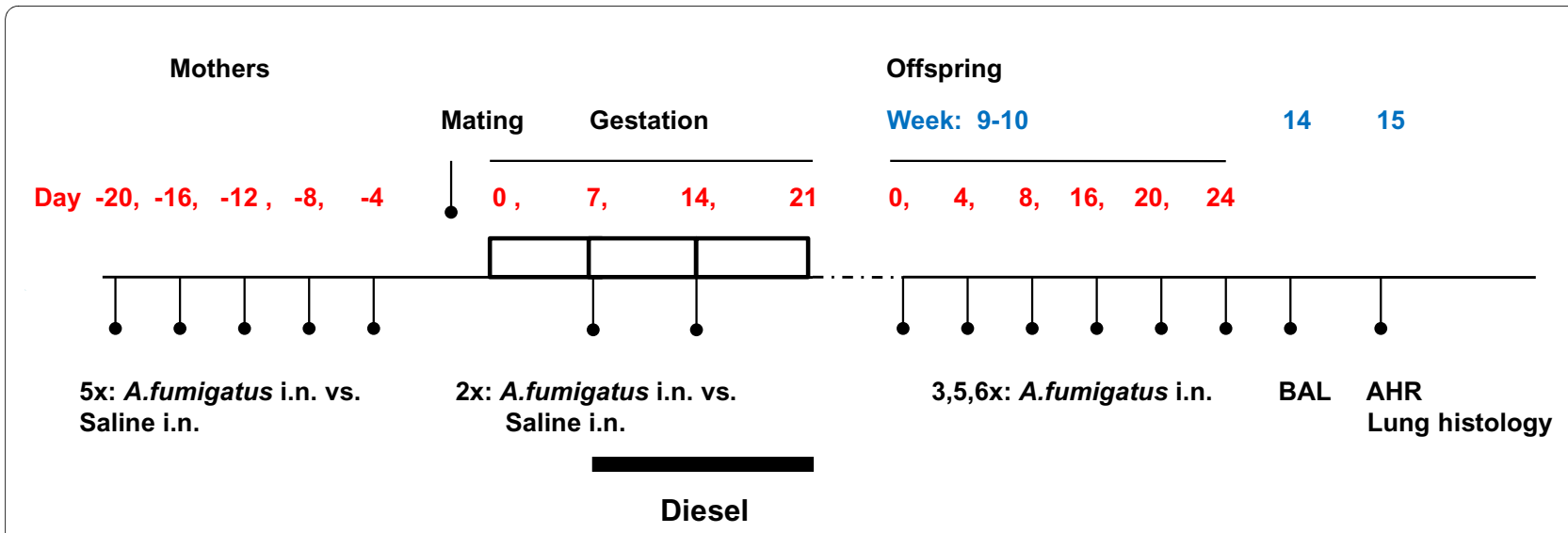

Figure 1 Experimental protocol. Adult females received 5 dosages of A. fumigatus or saline, 20,16,12,8, and 4 days prior to mating. During the second and third weeks of pregnancy, mothers received diesel exhaust particle exposure Monday through Friday plus A. fumigatus or saline on days 7 and 14. AHR: Airway hyperreactivity BAL: Bronchoalveolar lavage i.n: intranasal 3 x: 3 doses of A. fumigatus 5 x: 5 doses of A. fumigatus 6 x: 6 doses of A. fumigatus

each mouse with $1 \mathrm{ml}$ of phosphate buffered saline (PBS) 24 hours after the last allergen challenge. Lavage fluid was centrifuged at $4^{\circ} \mathrm{C} 1500 \mathrm{rpm}$ for 5 minutes. Cell pellets were resuspended in $1 \mathrm{ml}$ of phosphate buffered saline. Slides were prepared using a cytocentrifuge (Cytospin;Shandon) at $500 \mathrm{rpm}$ for 5 minutes then stained with Wright-Giemsa stain (Sigma-Aldrich, St. Louis, MO). 100 cells total were counted for each sample from 10 randomly chosen viewing fields and total eosinophil, lymphocyte, macrophage and neutrophil counts were quantified by a blinded reader.

\section{Airway hyperreactivity, lung histology and assessment for remodeling}

At median age 15.5 weeks, additional mice were anesthetized and intubated with a $20 \mathrm{~g}$ catheter inserted directly into the trachea via a neck dissection, then placed on a flexivent ventilator. A nebulizer attached to the flexivent apparatus exposed mice to increasing concentrations of methacholine at 8, 16, 32, 64 mg/ml (Sigma-Aldrich, St. Louis, MO). Airway resistance was determined by the flexivent-apparatus (SCIREQ, Montreal, Quebec, Canada) [18]. The shape of each dose-response curve was examined to determine whether each mouse responded to aerosolized methacholine, as described elsewhere [19]. Data obtained from aberrant curves were discarded prior to data analysis.

Immediately subsequent to the AHR testing, lungs were inflated and stored in $10 \%$ formalin. Lungs were paraffinembedded and sections were stained with hematoxylin and eosin (Sigma-Aldrich, St. Louis, MO). Under blinded conditions, each lung was scored for perivascular inflammation, peribronchial inflammation and arterial remodeling as previously described [13]. For perivascular and peribronchial inflammation, lungs were scored semi- quantitatively as follows: 1 = normal with very few inflammatory cells bordering the arteries or airways; $2=$ scattered inflammatory cells surrounding the artery or airway up to two rings in depth; 3 = cell cuffs or clusters of inflammatory cells surrounding the artery or airway three rings or more in depth. Arterial remodeling was scored as follows: $1=$ normal; $2=$ thickened vascular wall with intact lumen and circular media; $3=$ obstructed lumen and thickened wall lined with disorganized layers of cells.

\section{A. fumigatus-specific $T$ cell proliferation}

Splenocytes $\left(1 \times 10^{6} / \mathrm{ml}\right)$ were seeded in triplicate in 96 well plates and treated with A. fumigatus (Hollister-Stier, Spokane, WA) at $0,20 \mathrm{ug} / \mathrm{ml}$ or $40 \mathrm{ug} / \mathrm{ml}$ and CD3 $10 \mu \mathrm{g} /$ $\mathrm{ml}$ (BD, Franklin Lakes, NJ) and incubated with $5 \% \mathrm{CO}_{2}$ for five days at $37^{\circ} \mathrm{C} .{ }^{3} \mathrm{H}$-thymidine uptake was assessed on day 5 as described [20].

\section{Statistical analysis}

One-way analysis of variance (ANOVA) was used to compare mean differences across treatment groups followed by Tukey HSD test except where noted. Nonparametric rank order correlations were used to compare continuous data (eg. IgE levels and eosinophil counts) between treatment groups. Differences were considered statistically significant at $\mathrm{p}<0.05$.

\section{Results}

Effects of A. fumigatus, diesel exhaust exposure, on adult female pregnant mice

Adult female mice sensitized to A. fumigatus developed higher total IgE levels than those treated with only vehicle saline solution ( $\mathrm{p}<0.0001$, MannWhitney U, Figure 2). Higher eosinophil absolute counts and percentage of total 


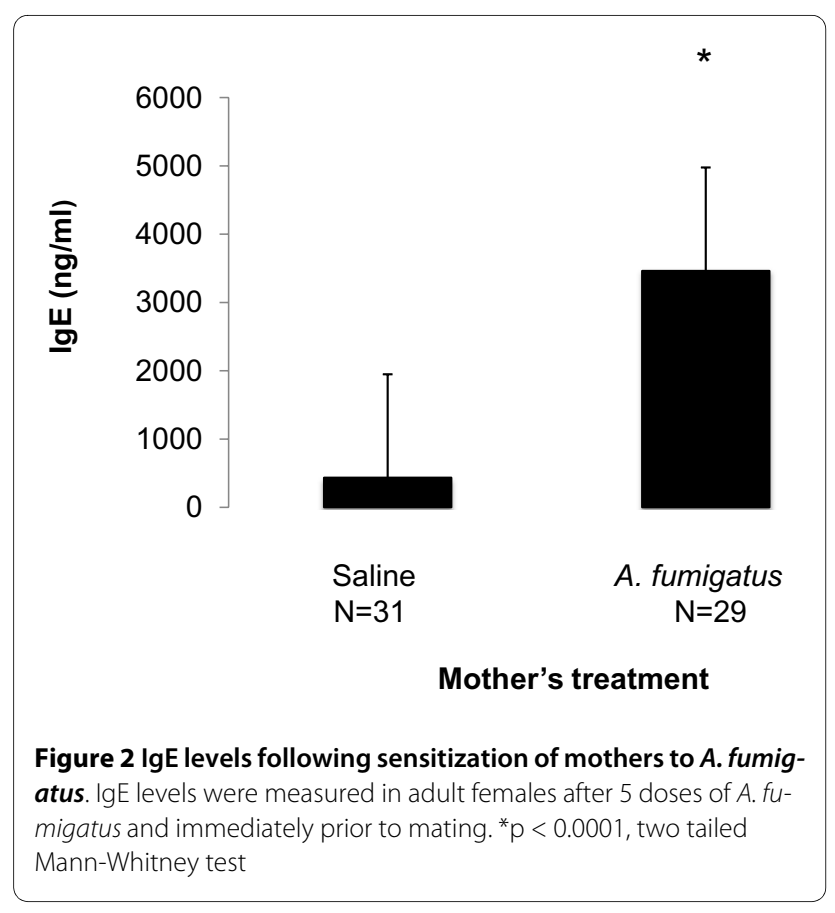

white blood cells also were detected among sensitized adult female mice immediately prior to euthanasia on bronchoalveolar lavage ( $\mathrm{p}<0.0001$ for both). There were no significant differences in airway inflammation across treatment groups following breeding. The airway inflammation score in retired female breeder mice treated with saline only (mean score: 1.7) was greater than the expected baseline of 1.0-1.4 observed in 2-3 month old experimental wild type mice [13,21]. To ascertain whether prenatal exposure to A. fumigatus and/or DEP also can induce airway remodeling in the mothers, as reported in wildtype C57BL/6 mice, pulmonary arterial remodeling was assessed across treatment groups [13]. Significant differences in arterial remodeling across groups were not detected.

\section{Ig induction in offspring after three, five and six doses of A. fumigatus}

Adult offspring from mothers who received $A$. fumigatus or DEP alone, or A. fumigatus and DEP together, developed lower levels of total IgE when assessed after the fifth dose of $A$. fumigatus compared to offspring from mothers treated with saline only prior to mating $(\mathrm{p}<0.0001$ ANOVA, Figure 3a). In addition, IgE levels from offspring from mice exposed to DEP alone were lower than those from offspring from mice exposed to A. fumigatus alone ( $\mathrm{p}<0.05$ Tukey HSD test). Adult offspring from mothers who received $A$. fumigatus or DEP alone, or DEP and $A$. fumigatus together, developed lower IgE levels compared with levels from offspring whose mothers received saline alone when assessed after the sixth dose of allergen treatment as well ( $\mathrm{p}<0.0001$ ANOVA). In addition, IgE levels from adult offspring of mice that were treated with DEP and $A$. fumigatus were lower than those from offspring of mice that were treated with A. fumigatus alone ( $\mathrm{p}<0.05$, Tukey HSD test). Significant differences in IgE levels were not apparent after the third dose of $A$. fumigatus

In contrast, offspring from mothers exposed to $A$. fumigatus, DEP, or both DEP and A. fumigatus, developed greater $I g G_{1}$ levels compared to offspring of mothers treated with saline. This effect was significant after the fifth and sixth, but not third, doses of A. fumigatus treatment ( $\mathrm{p}<0.001$ ANOVA, Figure 3b).

Further, offspring from mothers exposed to A. fumigatus, or both DEP and A. fumigatus, developed greater $\mathrm{IgG}_{2 \mathrm{a}}$ levels compared to offspring of mothers treated with saline alone when assessed after the fifth dose of $A$. fumigatus ( $\mathrm{p}<0.001$ ANOVA). Also, offspring from mothers exposed to both DEP and A. fumigatus, developed greater $\operatorname{IgG}_{2 \mathrm{a}}$ levels compared to offspring of mothers exposed to either DEP or A. fumigatus alone ( $\mathrm{p}<0.01$ Tukey HSD test). Adult offspring from mothers who received A. fumigatus alone, or DEP and A. fumigatus together, developed greater $\operatorname{IgG}_{2 \mathrm{a}}$ levels compared with levels from offspring whose mothers received saline or DEP alone after the sixth dose of allergen as well ( $\mathrm{p}<$ 0.0001 ANOVA). In contrast, after the third dose of $A$. fumigatus, a reduction in $\mathrm{IgG}_{2 \mathrm{a}}$ was detected among offspring from mice exposed to DEP compared with those treated with saline $(\mathrm{p}<0.05$, Tukey HSD, Figure 3c).

Prenatal exposure to A. fumigatus and diesel exhaust particles was associated with reduced airway eosinophilia in adult offspring

Adult offspring from mothers that received both $A$. fumigatus and DEP developed significantly less airway eosinophilia (mean eosinophil count $13.24 \pm 2.04 \%$ ) compared to offspring from mothers that had received $A$. fumigatus (26.44 $\pm 2.89 \%, \mathrm{p}=0.01$, Tukey HSD) or saline $(23.83 \pm 3.33 \%, \mathrm{p}=0.05$, Tukey HSD $)$ alone. The first result (A. fumigatus and DEP lower than A. fumigatus) was replicated when examining absolute numbers of eosinophils ( $\mathrm{p}<0.001$ on ANOVA and $\mathrm{p}<0.01$ by Tukey HSD). Adult offspring from mothers that received both A. fumigatus and DEP also developed higher levels of macrophage counts compared to offspring of mothers that had received A. fumigatus ( $\mathrm{p}=0.01$, Tukey HSD) or saline $(\mathrm{p}=0.05$, Tukey HSD) alone (Figure 4$)$. Airway eosinophil counts did not correlate with IgE levels measured at any of the time points (Spearman rank correlation R-value $=-0.055$ after the third dose, 0.019 after the fifth dose and -0.082 after the sixth dose, $\mathrm{p}=$ nonsignificant (NS) for each). Airway eosinophil counts also did not correlate with $\mathrm{IgG}_{1}$ levels after the fifth ( $\mathrm{R}$-value $=$ $0.216, \mathrm{p}=\mathrm{NS})$ or sixth dose $(\mathrm{R}$-value $=-0.185, \mathrm{p}=\mathrm{NS})$. 



\section{Saline \\ Diesel \\ A.fumigatus \\ Diesel+A.fumigatus \\ Mother's treatment}

Figure 3 lg induction in offspring after three, five and six doses of A. fumigatus. a) lgE was reduced after the fifth and sixth ( $p<0.0001$ on ANO$V A$ ), but not third ( $p=N S, A N O V A$ ), doses among offspring mice whose mothers were exposed to either $A$. fumigatus or diesel exhaust particles or both. ${ }^{*} p<0.01$, when compared to saline alone by Tukey HSD. $+p<0.05$, when compared to A. fumigatus alone by Tukey HSD b) IgG 1 was greater after the fifth, sixth ( $p<0.0001$ on ANOVA), but not third ( $p=N S$, ANOVA), doses among mice whose mothers were exposed to either $A$. fumigatus or diesel exhaust particles or both. ${ }^{*} p<0.01$, when compared to saline alone by Tukey HSD. $+p<0.05$, when compared to saline alone by Tukey HSD. c) IgG2a was greater after the fifth, sixth ( $p<0.0001$ on ANOVA) dose among mice whose mothers were exposed to A. fumigatus or diesel exhaust particles plus A. fumigatus. ${ }^{*} p<0.01$, when compared to saline alone by Tukey HSD. Levels also were greater among offspring of mothers that were exposed to both diesel exhaust particles and A. fumigatus when compared to offspring of mothers treated either with diesel exhaust or A. fumigatus alone, $p<0.01$. $\uparrow p<0.01$, when compared to diesel exhaust particles alone by Tukey HSD. $\neq p<0.05$ on ANOVA and when compared to saline alone by Tukey HSD. Sample sizes corresponding to the figures vary as follows: Saline 11-14; Diesel 11-15; A. fumigatus: 8-18; Diesel and A. fumigatus 13-25. 


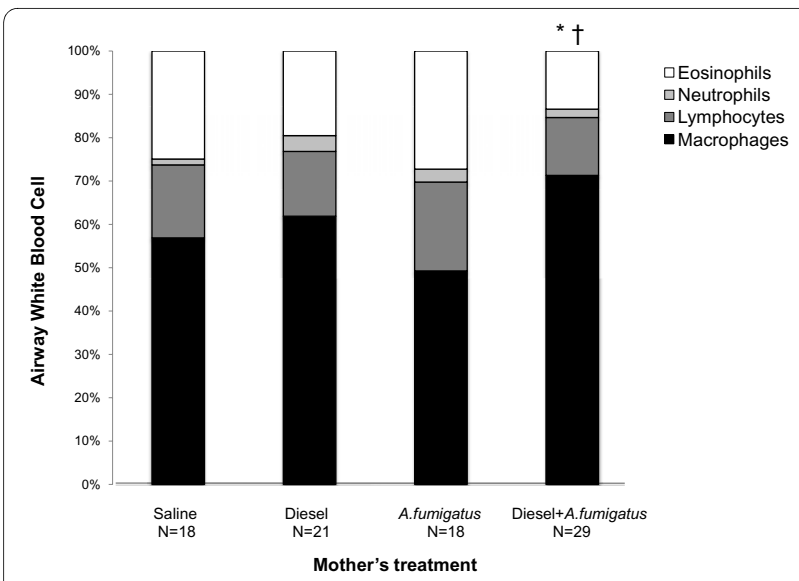

Figure 4 Differential airway cell counts in offspring after five and six doses of $\boldsymbol{A}$. fumigatus. Eosinophil counts were significantly decreased (and macrophages significantly increased) among offspring from mothers following diesel exhaust and $A$. fumigatus compared to offspring of mothers treated with saline alone, ${ }^{*} p<0.0002$ on ANOVA and $p<0.05$ by Tukey HSD or with A. fumigatus alone, $\uparrow p<0.0003$ on ANOVA and $p<0.01$ by Tukey HSD.

Effects on perivascular, peribronchial airway inflammation, airway remodeling, and airway hyperreactivity

To ascertain whether systemic changes in Ig levels and airway changes in eosinophil counts were associated with additional histological and physiological alterations, perivascular, peribronchial airway inflammation, airway remodeling, and airway hyperreactivity were assessed in the adult offspring following prenatal exposure to diesel exhaust and/or A. fumigatus. On histological examination, examples of perivascular and peribronchial inflammation and arterial muscularization were detected among adult offspring of mice exposed to A. fumigatus when compared to offspring of mice exposed to saline (Figure 5). Differences were not observed among offspring from mice that were exposed to DEP, when compared to offspring of mice that received saline, or among offspring of mice that received DEP and A. fumigatus, compared to $A$. fumigatus alone.

However, using an established semi-quantitative scoring system [13], only a nonsignificant trend in airway inflammation was observed among mice that were treated with $A$. fumigatus when compared to mice whose mothers were treated with saline alone $(1.84 \pm 3.74$ saline vs. $2.05 \pm 0.07$ A. fumigatus mean airway inflammation score $\pm \mathrm{SE}, \mathrm{p}=0.11$ ANOVA)(Figure 6a). Also, we were unable to detect a correlation between total airway inflammation scores and IgE levels measured at any of the 3 time points ( $\mathrm{R}$-value $=0.096$ after the third dose, 0.016 after the fifth dose and -0.077 after the sixth dose, $p$ = NS for each) (Figure 6b). Correlations between inflammation score and $\operatorname{IgG}_{1}(\mathrm{R}$-value $=0.228$ after the fifth

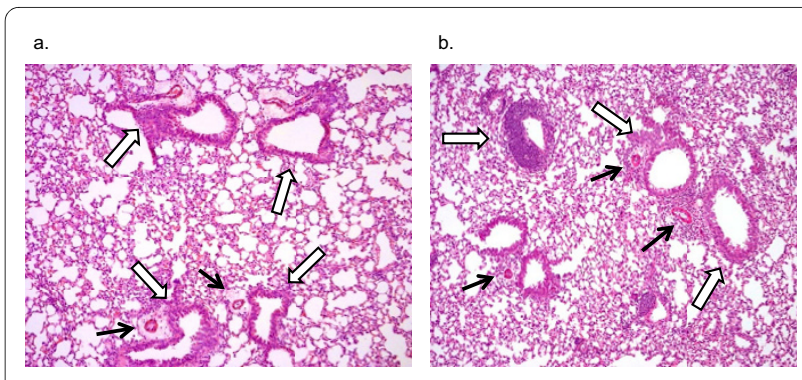

Figure 5 Histological changes in offspring from mothers exposed to saline or A. fumigatus. a.) Representative histology from lungs of offspring whose mother was treated with saline following 5 doses of $A$. fumigatus starting at 9-10 weeks. The photomicrograph was taken from a lung section stained with Hematoxylin and Eosin. White arrows point to perivascular and peribronchial inflammation, black arrowheads point to mild arterial muscularization. b.) Representative histology from lung of offspring whose mother was treated with $A$. fumigatus following 5 doses of A. fumigatus starting at 9-10 weeks. The photomicrograph was taken from a lung section stained with Hematoxylin and Eosin. White arrows point to perivascular and peribronchial inflammation, black arrowheads point to arterial muscularization. No differences were observed among offspring of mice that received diesel exhaust, particles when compared to offspring of mice that received saline. No differences were observed among offspring of mice that received diesel and A. fumigatus, compared to A. fumigatus alone.

dose, 0.222 after the sixth dose, $\mathrm{p}=\mathrm{NS}$ for each) were not detected either.

To ascertain whether prenatal exposure to $A$. fumigatus and/or DEP can induce airway remodeling in adult sensitized offspring, pulmonary arterial remodeling was assessed in the mice offspring. Offspring from mothers who received $A$. fumigatus and/or DEP during pregnancy did not exhibit significant differences in the degree of arterial airway remodeling compared to offspring of mothers who received saline (Figure 6b). Arterial remodeling scores between mothers and their offspring exhibited a borderline correlation (spearman $\mathrm{R}=0.269, \mathrm{p}=$ 0.096).

In addition, differences in AHR across any treatment groups were absent (data not shown).

\section{Prenatal exposure to A. fumigatus, diesel was not associated with altered $A$. fumigatus-induced $T$ cell proliferation}

To determine whether prenatal exposure to A. fumigatus and/or DEP would be associated with altered antigenspecific $\mathrm{T}$ cell proliferation in the offspring, splenocytes were tested following induction with several doses of $A$. fumigatus or anti-CD3. We found that antigen-specific proliferation in the adult offspring was not affected by $A$. fumigatus or DEP exposure administration to the mother (data not shown). 


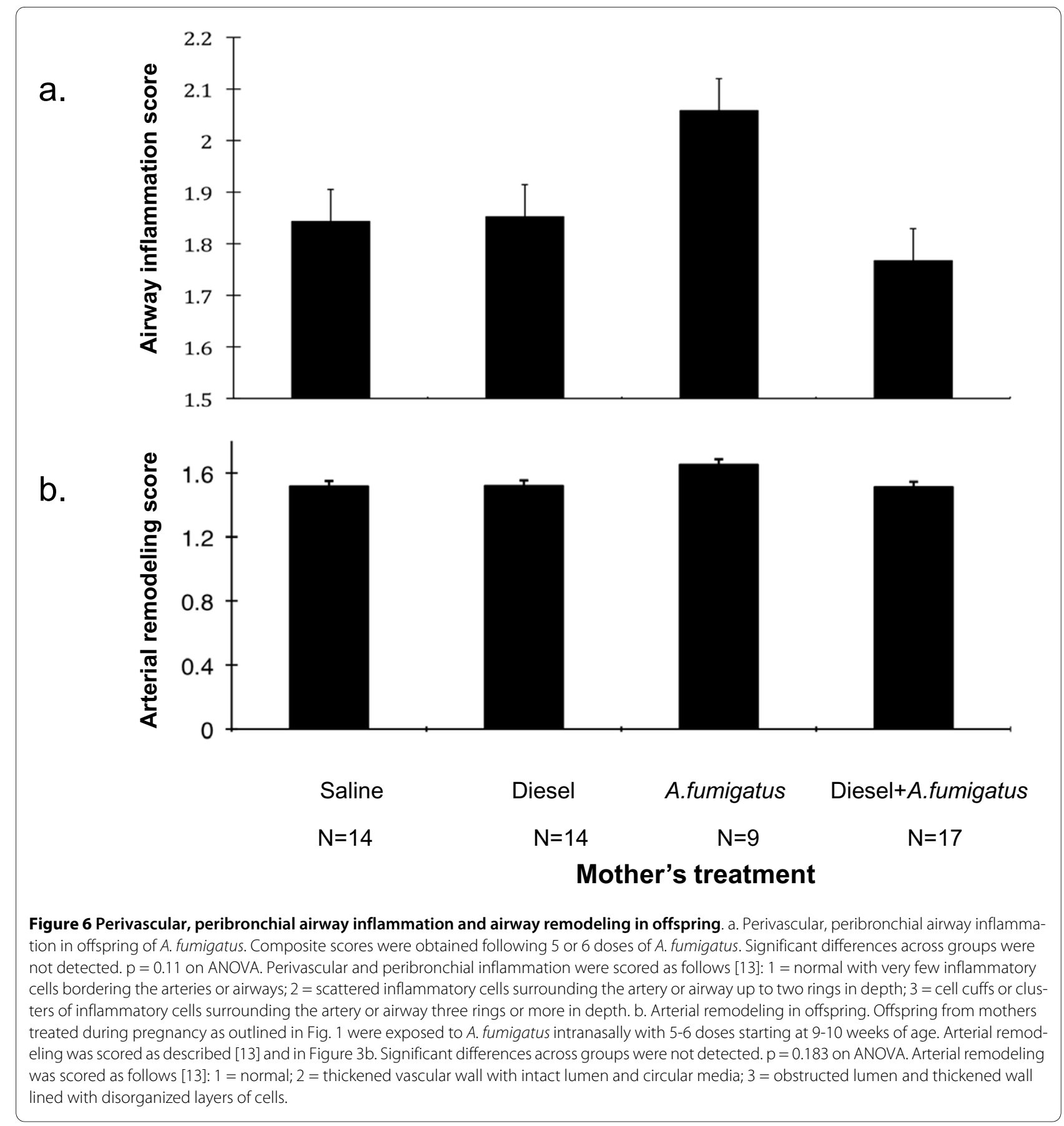

\section{Discussion}

These results suggest that exposures to A. fumigatus prior to and during pregnancy were associated with diminished IgE production and airway eosinophilia. The latter occurred following prenatal exposure to both $A$. fumigatus and diesel. The parallel increases in IgG levels suggest that the antibody responses were specific to IgE. These findings indicate that prenatal exposure to $A$. fumigatus, may be associated with protection from sys- temic and airway allergic immune responses in adult offspring.

While these results may appear contradictory to several studies that show prenatal sensitization (i.e. to ovalbu$\mathrm{min}$ ) is associated with greater allergic immune responses in the offspring [5], they are consistent with a few studies that suggest prenatal environmental exposures can suppress the subsequent risk for an asthma-related phenotype or induce tolerance. For example, transfer of antigens from mother to mouse pup via breast milk has 
induced oral tolerance and antigen-specific protection from allergic airway disease [22]. In addition, prenatal sensitization to $D$. pteroynissinus was associated with lower total and D. pteroynissinus-IgE levels in the offspring. Similar to our model, exposure to allergen prior to mating reduced allergen sensitization in the offspring at the humoral level [10]. In more recent work by the same group, the induction of allergic sensitization versus tolerance following prenatal exposure to ovalbumin was determined to be dependent on the dose and timing of exposure. Specifically, prenatal oral exposure to high dose ovalbumin was associated with lower ovalbumin-IgE in the pups at age 3 days following immunization. The effect was transient, and subsequent increases in ovalbuminIgE levels were detected at age 25 days. Also, the effect was best observed if the ovalbumin treatments occurred during the first week of pregnancy. However, pups born to mothers who received prenatal oral administration of low dose ovalbumin showed similar decreases in ovalbumin IgE levels and antigen-specific $\mathrm{T}$ cell proliferation, but this tolerogenic effect was more sustained[23]. Prenatal LPS exposure also was associated with suppression of $\mathrm{IgG}_{1}$ and IgE and reduction of interleukin (IL)-5 and IL13 in splenic mononuclear cells [7]. Besides endotoxin, prenatal oral exposure to the chemical bisphenol A has been associated with preferential $\mathrm{T}$ helper (Th) 1 immune responses in sensitized adult offspring mice [24]. Hence, in the model described here, prenatal exposure to A. fumigatus and diesel may have timed or dosed so as to favor establishment of tolerance instead of allergic sensitization.

While postnatal exposure to mold has been associated with greater asthma severity or emergency room visits for asthma [24-27], recent studies suggest that exposure to mold allergen after birth may be protective. These include two cross sectional cohort studies that found higher levels of fungal $\beta(1,3)$-glucans, fungal extracellular polysaccharides and endotoxin in dust collected from mattresses used by asymptomatic children age 5-13 compared with those used by atopic children who wheezed $[28,29]$. It has been hypothesized that fungal products besides the associated allergens, such as dust endotoxin, extracellular polysaccharides (EPS) and glucans may induce immunologic protection from the development of atopic disease $[28,29]$. As another example, inner-city children, aged 2 to 6 years old, living in homes with either comparatively low $(<2 \mu \mathrm{g} / \mathrm{g}$ Mus m 1$)$ or high $(>29.9 \mu \mathrm{g} /$ g Mus $m$ 1) dust levels of mouse allergen developed attenuated humoral responses in comparison to those who lived in homes with a medium level of measured allergen in their dust (2-7.9 $\mu \mathrm{g} / \mathrm{g}$ Mus $\mathrm{m} \mathrm{1)}$. This work also suggests that the development of protection from allergic sensitization occurs and may be related to the dose of allergen exposure. However, the extent of allergic sensiti- zation, rather than the measured level of allergen detected in dust or delivered via aerosol, tends to be more strongly associated with allergy symptoms in an innercity cohort study [30].

EPA has estimated occupational DEP exposures to range from $39-191 \mu \mathrm{g} / \mathrm{m}^{3}$ for railroad workers, $4-748$ $\mu \mathrm{g} / \mathrm{m}^{3}$ for firefighters, and $7-98 \mu \mathrm{g} / \mathrm{m}^{3}$ for public transit workers and airport crews [31]. So while the chronic administration of inhaled diesel exhaust particles may have mimicked some natural physiological conditions in this model, the levels employed are higher than most urban environments and some occupational ones. Diesel exposure has been associated with upregulation of the allergic immune responses and airway remodeling in both animal and human studies [32,33]. However, the independent and synergistic effects of prenatal diesel exposure administered in this manner and reported here seem small. Adult offspring from mothers who received DEP alone, or A. fumigatus and DEP together, developed lower levels of total IgE, and greater levels of $\operatorname{IgG}_{1}$, when assessed after the fifth and sixth dose of allergen. Paradoxically, after the third dose of $A$. fumigatus, a reduction in $\mathrm{IgG}_{2 \mathrm{a}}$ was detected among offspring from mice exposed to DEP compared with those treated with saline. Also, adult offspring of mothers that received both $A$. fumigatus and DEP developed significantly less airway eosinophilia compared to offspring of mothers that had received $A$. fumigatus alone. Combined, these results suggest that prenatal DEP exposure independently may have conferred some protection against allergic immune responses in the adult offspring in this model. These findings were unexpected, especially given previous research using the engine byproduct residual oil fly ash as the air pollutant that induced greater airway eosinophilia and hyperreactivity in the OVA-sensitized offspring [2]. It is unclear whether the disparate phenotypes are related to the antigens administered (ovalbumin vs. A. fumigatus), components and dose of the air pollutants, strain of mouse, or age of the offspring following allergen sensitization (less than 5 weeks vs. 9-10 weeks).

Associations between prenatal exposure to DEP and/or A. fumigatus and airway arterial remodeling in adult offspring were not statistically significant, with only mild changes detected during histological examination. Exposure to A. fumigatus has been shown to exacerbate an asthma phenotype in rats by aggravating Th2 inflammation, increasing AHR, and inducing airway remodeling [34]. Previously, a few mouse models have induced airway remodeling following repeated and chronic OVA exposure and the recruitment of eosinophils, IL-13 and profibrotic cytokines have been implicated [35-37]. Our group previously showed that adult C57BL/ 6 mice treated intermittently with $A$. fumigatus for a prolonged period of 
time developed remodeling of small to medium sized pulmonary arteries [13]. In another mouse model, maternal exposure to cigarette smoke during pregnancy was found to be associated with airway remodeling in the offspring at ten weeks of age, as demonstrated by increases in airway smooth muscle thickness, collagen deposition and house dust mite induced increases in neutrophils, mast cells and goblet cell hyperplasia [38]. From a cohort study, offspring of mothers who smoked during pregnancy developed permanent vascular damage that was not apparent in offspring of non-smoking mothers [39]. This current study, to our knowledge, represents the first examination of the effects of these environmental exposures on airway remodeling across generations of mice.

Several plausible mechanisms may explain how prenatal exposures may help modulate the development of allergic and/or airway immune response in the offspring. It has been reported that antigen-specific $\mathrm{T}$ cell and $\mathrm{B}$ cell immune responses in the fetus can occur distinctly from those of the mother, as demonstrated by our group in response to vaccination against influenza [40]. In addition, previous reports also suggested that the transfer of allergy across the placenta may be regulated by the transfer of cytokines that may influence the development of allergic sensitization. Supportive data include a murine model that demonstrates that administration of anti-IL-4 can inhibit allergic immune responses from sensitization to OVA in the offspring [41]. In addition, combined inhaled diesel exhaust and A. fumigatus exposure has been shown to induce hypermethylation of multiple CpG sites of the interferon-gamma (IFNg) promoter and hypomethylation of one $\mathrm{CpG}$ site of the IL-4 promoter with associated changes in IgE levels, suggestive of the contribution of epigenetic regulation following environmental exposures [16]. These mechanisms seem plausible in light of recent associations between prenatal exposure to polycyclic aromatic hydrocarbons or high methyl diet and DNA methylation of asthma candidate genes [6,42]. However, these reports do not directly explain mechanistically how prenatal exposure to A. fumigatus, or diesel, may induce protection from allergy in the offspring, especially in light of past data that suggest A. fumigatus induces greater, not repressed, Th2 cytokine production [13]. In one study, offspring of Balb/c mice whose mothers were tolerized with ovalbumin by means of oral application of antigen also were protected from the development of an asthma-like phenotype as late as 8 month after birth. This protection was blocked by inhibition of IFN $\gamma$ [42]. Transfer of IgG antibodies from suckling or from the placenta has also been shown to suppress IgE following prenatal exposures to egg albumin [22]. Rats whose mothers were immunized with egg albumin during pregnancy experienced a diminished capacity to develop IgE and enhanced IgG responses during early adulthood, and these results were replicated when separate offspring were administered small quantities of immune serum 3 weeks after birth[43].

Some limitations of this study merit discussion. First, we used only one strain of mice to obtain the above findings even though it has been shown that when comparing acute injury responses, such as airway remodeling, patterns are unique to different strains[44]. Also, the use of a mouse model does not give us a comprehensive representation of what occurs after prenatal sensitization in humans because we are not able to accurately replicate some human behaviors such as smoking and diet. Relatively higher inflammation scores among retired mothers and their adult offspring are difficult to explain, but could be a result of accumulated lung injury due to dust from dirty bedding in breeder cages or stress (personal observation).

\section{Conclusion}

In conclusion, our results indicate that A. fumigatus administration during pregnancy resulted in protection from systemic and airway allergic responses. Prenatal diesel exhaust particle exposure also was associated with reduced IgE levels and suppressed airway eosinophilia in the adult offspring. These results suggest that prenatal environmental exposures can induce exert systemic and airway immune changes in the adult offspring related to respiratory disease. These results highlight the need to consider the health effects of prenatal exposures on offspring, even through adulthood.

\section{List of abbreviations}

AHR: airway hyperreactivity; AKP: alkaline-phosphatase; BAL: bronchoalveolar lavage; BHR: bronchial hyperresponsiveness; DEP: diesel exhaust particles; EPS: extracellular polysaccharides; ETS: environmental tobacco smoke; HDM: house dust mite; IFN: interferon; Ig: immunoglobulin; IL: interleukin; in: intranasal; LPS: lipopolysaccharide; NS: non-significant; NYU: New York University; OVA: ovalbumin; $\mathrm{PAH}$ : polycyclic aromatic hydrocarbon; PMA: phorbol 12-myristate 13-acetate; RUNX3: Runt-related transcription factor 3; Th: T-helper

\section{Competing interests}

The authors declare that they have no competing interests.

\section{Authors' contributions}

$\mathrm{LL}$ carried out the experimental work, performed some of the statistical work, and drafted the manuscript. HZ carried out the experimental work. CQ carried out all exposure related experiments. GG participated in the design of the study and advised on the experimental work. MB carried out a significant proportion of the experimental work. XJ worked with CQ administer the diesel exposure. FPP participated in the design of the study and advised on the experimental work. PHF participated in the design of the study and advised on the experimental work. LCC participated in the design of the study and advised on the experimental work. RLM conceived of the study, performed the statistical work, and participated in its design and coordination and drafted the manuscript. All authors read and approved the final manuscript. 


\section{Acknowledgements}

The authors thank Eleen Daley and Eun Soo Kwak, for technical assistance. This work was supported by the National Institute of Health R21ES013063, P30 ES009089, P01 ES09600, PO1-E HL071042, HL066211, HL079094, P50 ES 015905, S00260, RO1-ES015495; Environmental Protection Agency EPA 827027

\section{Author Details}

1Division of Pulmonary, Allergy and Critical Care Medicine, Department of Medicine, Columbia University College of Physicians and Surgeons, New York, New York 10032, USA, ${ }^{2}$ Environmental Health Sciences, New York University, Tuxedo, New York 10987, USA and ${ }^{3}$ Columbia Center for Children's Environmental Health, Mailman School of Public Health Columbia University, New York, New York 10032, USA

Received: 21 December 2009 Accepted: 11 May 2010 Published: 11 May 2010

\section{References}

1. Miller RL, Garfinkel R, Horton M, Camann D, Perera FP, Whyatt RM, Kinney PL: Polycyclic aromatic hydrocarbons, environmental tobacco smoke, and respiratory symptoms in an inner-city birth cohort. Chest 2004, 126:1071-1078

2. Hamada K, Suzaki Y, Leme A, Ito T, Miyamoto K, Kobzik L, Kimura H: Exposure of pregnant mice to an air pollutant aerosol increases asthma susceptibility in offspring. J Toxicol Environ Health A 2007, 70:688-695.

3. Moshammer H, Bartonova A, Hanke W, Hazel P van den, Koppe JG, Kramer U, Ronchetti R, Sram RJ, Wallis M, Wallner P, Zuurbier M: Air pollution: A threat to the health of our children. Acta Paediatr Suppl 2006, 95:93-105.

4. Jedrychowski W, Flak E: Maternal smoking during pregnancy and postnatal exposure to environmental tobacco smoke as predisposition factors to acute respiratory infections. Environ Health Perspect 1997, 105:302-306.

5. Fedulov AV, Leme A, Yang Z, Dahl M, Lim R, Mariani TJ, Kobzik L: Pulmonary exposure to particles during pregnancy causes increased neonatal asthma susceptibility. Am J Respir Cell Mol Biol 2008, 38:57-67.

6. Hollingsworth JW, Maruoka S, Boon K, Garantziotis S, Li Z, Tomfohr J, Bailey N, Potts EN, Whitehead G, Brass DM, Schwartz DA: In utero supplementation with methyl donors enhances allergic airway disease in mice. J Clin Invest 2008, 118:3462-3469.

7. Blumer N, Herz U, Wegmann M, Renz H: Prenatal lipopolysaccharideexposure prevents allergic sensitization and airway inflammation, but not airway responsiveness in a murine model of experimental asthma. Clin Exp Allergy 2005, 35:397-402.

8. Gerhold K, Avagyan A, Seib C, Frei R, Steinle J, Ahrens B, Dittrich AM, Blumchen K, Lauener R, Hamelmann E: Prenatal initiation of endotoxin airway exposure prevents subsequent allergen-induced sensitization and airway inflammation in mice. J Allergy Clin Immunol 2006, 118:666-673.

9. Douwes J, Cheng S, Travier N, Cohet C, Niesink A, McKenzie J, Cunningham C, Le Gros G, von Mutius E, Pearce N: Farm exposure in utero may protect against asthma, hay fever and eczema. Eur Respir $\rfloor$ 2008, 32:603-611.

10. Fusaro AE, Maciel M, Victor JR, Oliveira CR, Duarte AJ, Sato MN: Influence of maternal murine immunization with dermatophagoides pteronyssinus extract on the type I hypersensitivity response in offspring. Int Arch Allergy Immunol 2002, 127:208-216.

11. Patel MM, Miller RL: Air pollution and childhood asthma: Recent advances and future directions. Current Opinions in Pediatrics 2009, 21:235-242.

12. Wang J, Visness CM, Calatroni A, Gergen PJ, Mitchell HE, Sampson HA: Effect of environmental allergen sensitization on asthma morbidity in inner-city asthmatic children. Clin Exp Allergy 2009, 39(9):1381-89.

13. Daley E, Emson C, Guignabert C, de Waal Malefyt R, Louten J, Kurup VP, Hogaboam C, Taraseviciene-Stewart L, Voelkel NF, Rabinovitch M, Grunig E, Grunig G: Pulmonary arterial remodeling induced by a Th2 immune response. J Exp Med 2008, 205:361-372.

14. Liu J, Ballaney M, Al-alem U, Quan C, Jin X, Perera F, Chen LC, Miller RL: Combined inhaled diesel exhaust particles and allergen exposure alter methylation of $t$ helper genes and IgE production in vivo. Toxicological Sciences 2008, 102:76-81.
15. McDonald JD, Barr EB, White RK: Design, characterization, and evaluation of a small-scale diesel exhaust exposure system. Aerosol Science and Technology 2004, 38:62-78.

16. Liu J, Ballaney M, Al-alem U, Quan C, Jin X, Perera F, Chen LC, Miller RL: Combined inhaled diesel exhaust particles and allergen exposure alter methylation of thelper genes and ige production in vivo. Toxicol $\mathrm{SCi}$ 2008, 102:76-81.

17. Matson AP, Zhu L, Lingenheld EG, Schramm CM, Clark RB, Selander DM, Thrall RS, Breen E, Puddington $L$ : Maternal transmission of resistance to development of allergic airway disease. J Immunol 2007, 179:1282-1291.

18. Oldenburg PJ, Wyatt TA, Factor PH, Sisson JH: Alcohol feeding blocks methacholine-induced airway responsiveness in mice. Am J Physiol Lung Cell Mol Physiol 2008, 296(1):L109-14.

19. Lu FL, Johnston RA, Flynt L, Theman TA, Terry RD, Schwartzman IN, Lee A Shore SA: Increased pulmonary responses to acute ozone exposure in obese db/db mice. Am J Physiol Lung Cell Mol Physiol 2006, 290:L856-865.

20. Padula SJ, Lingenheld EG, Stabach PR, Chou CH, Kono DH, Clark RB: Identification of encephalitogenic $v$ beta-4-bearing $T$ cells in sjl mice. Further evidence for the $v$ region disease hypothesis? J Immunol 1991, 146:879-883.

21. Sur S, Wild JS, Choudhury BK, Sur N, Alam R, Klinman DM: Long term prevention of allergic lung inflammation in a mouse model of asthma by CpG oligodeoxynucleotides. J Immuno/ 1999, 162:6284-6293.

22. Verhasselt V, Milcent V, Cazareth J, Kanda A, Fleury S, Dombrowicz D, Glaichenhaus N, Julia V: Breast milk-mediated transfer of an antigen induces tolerance and protection from allergic asthma. Nat Med 2008 14:170-175

23. Fusaro AE, Brito CAd, Taniguchi EF, Muniz BP, Victor JR, Orii NM, Duarte AJdS, Sato MN: Balance between early life tolerance and sensitization in allergy: Dependence on the timing and intensity of prenatal and postnatal allergen exposure of the mother. Immunology 2009, 128:

24. Yoshino S, Yamaki K, Li X, Sai T, Yanagisawa R, Takano H, Taneda S, Hayashi $\mathrm{H}$, Mori Y: Prenatal exposure to bisphenol a up-regulates immune responses, including T helper 1 and thelper 2 responses, in mice. Immunology 2004, 112:489-495.

25. Inal A, Karakoc GB, Altintas DU, Pinar M, Ceter T, Yilmaz M, Kendirli SG: Effect of outdoor fungus concentrations on symptom severity of children with asthma and/or rhinitis monosensitized to molds. Asian Pac J Allergy Immunol 2008, 26:11-17.

26. Harley KG, Macher JM, Lipsett M, Duramad P, Holland NT, Prager SS, Ferber $J$, Bradman A, Eskenazi B, Tager IB: Fungi and pollen exposure in the first months of life and risk of early childhood wheezing. Thorax 2009, 64:353-358.

27. Hagmolen of Ten Have W, Berg NJ van den, Palen J van der, van Aalderen WM, Bindels PJ: Residential exposure to mould and dampness is associated with adverse respiratory health. Clin Exp Allergy 2007, 37:1827-1832

28. Schram-Bijkerk D, Doekes G, Boeve M, Douwes J, Riedler J, Ublagger E, von Mutius E, Benz M, Pershagen G, Wickman M, Alfven T, Braun-Fahrlander C, Waser M, Brunekreef B: Exposure to microbial components and allergens in population studies: A comparison of two house dust collection methods applied by participants and fieldworkers. Indoor Air 2006, 16:414-425.

29. Schram-Bijkerk D, Doekes G, Douwes J, Boeve M, Riedler J, Ublagger E, von Mutius E, Benz MR, Pershagen G, van Hage M, Scheynius A, BraunFahrlander C, Waser M, Brunekreef B: Bacterial and fungal agents in house dust and wheeze in children: The parsifal study. Clin Exp Allergy 2005, 35:1272-1278

30. Matsui EC, Eggleston PA, Breysse PN, Rand CS, Diette GB: Mouse allergenspecific antibody responses in inner-city children with asthma. J Allergy Clin Immunol 2007, 119:910-915.

31. EPA US: Health assessment document for diesel engine exhaust. Washington, DC, 600/8-90/057F 2002

32. Nordling E, Berglind N, Melén E, Emenius G, Hallberg J, Nyberg F, Pershagen G, Svartengren M, Wickman M, Bellander T: Traffic-related air pollution and childhood respiratory symptoms, function and allergies. Epidemiology 2008, 19:401-408

33. Dai J, Xie C, Vincent R, Churg A: Air pollution particles produce airway wall remodeling in rat tracheal explants. Am J Respir Cell Mol Biol 2003, 29:352-358. 
34. Gao FS, Qiao JO, Zhang Y, Jin XQ: Chronic intranasal administration of aspergillus fumigatus spores leads to aggravation of airway inflammation and remodelling in asthmatic rats. Respirology 2009, 14(3):360-370

35. Temelkovski J, Hogan SP, Shepherd DP, Foster PS, Kumar RK: An improved murine model of asthma: Selective airway inflammation, epithelial lesions and increased methacholine responsiveness following chronic exposure to aerosolised allergen. Thorax 1998, 53:849-856.

36. Leigh R, Ellis R, Wattie J, Southam DS, De Hoogh M, Gauldie J, O'Byrne PM, Inman MD: Dysfunction and remodeling of the mouse airway persist after resolution of acute allergen-induced airway inflammation. Am J Respir Cell Mol Biol 2002, 27:526-535.

37. Fulkerson PC, Rothenberg ME, Hogan SP: Building a better mouse model: Experimental models of chronic asthma. Clin Exp Allergy 2005, 35:1251-1253.

38. Blacquiere MJ, Timens W, Melgert BN, Geerlings M, Postma DS, Hylkema $M N$ : Maternal smoking during pregnancy induces airway remodeling in mice offspring. Eur Respir J 2009, 33(5):1131-40.

39. Geerts CC, Bots ML, Grobbee DE, Uiterwaal CS: Parental smoking and vascular damage in young adult offspring: Is early life exposure critical? The atherosclerosis risk in young adults study. Arterioscler Thromb Vasc Biol 2008, 28:2296-2302.

40. Rastogi D, Wang C, Mao X, Lendor C, Rothman PB, Miller RL: Antigenspecific immune responses to influenza vaccine in utero. $J$ Clin Invest 2007, 117:1637-1646

41. Hamada K, Suzaki Y, Goldman A, Ning YY, Goldsmith C, Palecanda A, Coull B, Hubeau C, Kobzik L: Allergen-independent maternal transmission of asthma susceptibility. J Immunol 2003, 170:1683-1689.

42. Frederica Perera W-yT, Herbstman Julie, Tang Dellang, Levin Linda, Miller Rachel, Shuk-mei Ho: Relation of DNA methylation of 59-CpG island of ACSL3 to transplacental exposure to airborne polycyclic aromatic hydrocarbons and childhood asthma. PLOS 2009, 4(2):e4488. doi:10.1371/journal.pone.0004488

43. Jarrett $\mathrm{EE}$, Hall $\mathrm{E}$ : IgE suppression by maternal IgG. Immunology 1983 48:49-58.

44. Kips JC, Anderson GP, Fredberg JJ, Herz U, Inman MD, Jordana M, Kemeny DM, Lotvall J, Pauwels RA, Plopper CG, Schmidt D, Sterk PJ, Van Oosterhout AJ, Vargaftig BB, Chung KF: Murine models of asthma. Eur Respir J 2003, 22:374-382.

doi: 10.1186/1710-1492-6-7

Cite this article as: Corson et al., Prenatal allergen and diesel exhaust exposure and their effects on allergy in adult offspring mice Allergy, Asthma \& Clinical Immunology 2010, 6:7

Submit your next manuscript to BioMed Centra and take full advantage of:

- Convenient online submission

- Thorough peer review

- No space constraints or color figure charges

- Immediate publication on acceptance

- Inclusion in PubMed, CAS, Scopus and Google Scholar

- Research which is freely available for redistribution

Submit your manuscript at www.biomedcentral.com/submit
C Biomed Central 